\title{
Design and Implementation of Intelligent Campus Support Service Platform Based on Cloud Computing
}

\author{
Wang Fangzheng \\ Jiangsu Vocational College of Medical, Yancheng 224005, China \\ wfz4393@163.com
}

Keywords: Intelligent campus, Supporting platform, Cloud computing

\begin{abstract}
With the continuous progress of the society, the requirements for the construction of educational informatization in colleges and universities are becoming higher and higher. In the face of the increasingly complex application needs of the intelligent campus, this paper briefly expounds the existing problems and the present situation of the research on educational informatization. The design and implementation of the intelligent campus support service platform of cloud computing are put forward, and the problems urgently needed to be solved are discussed.
\end{abstract}

\section{Introduction}

With the rapid development of Internet technology, great changes have been made in the informationization of university education. There are some problems in the construction of digital campus. It is difficult to manage and share information. The time and order of input information are not the same, which leads to the repeated input of some information and even to different systems. The same information will appear in different management systems, if the information needs to be changed or deleted, there will be problems. The utilization rate of resources is low: schools have different management systems, such as educational administration system, financial management system, information management system and so on. According to the survey, the utilization rate of these systems is extremely low, and the utilization rate of some systems is even less than ten percent. The maintenance of equipment management is difficult: the time and type of each system are variable, the warranty period of each equipment is different, and the utilization ratio of each equipment is also different, which makes the maintenance of equipment very difficult.

These problems make education informatization have to speed up the pace of progress, education informatization is in urgent need of transformation from the construction of digital campus to the construction of intelligent campus. Zhejiang University, Southwest University and Nanjing University of posts and Telecommunications have put forward the construction of intelligent campus, which is based on cloud computing and the Internet to realize the integration of campus information, the collection and transportation of information, the classification and storage of information. The push of information and the corresponding intelligent processing, realize each system of university, each department, connect each other, integrate information, and then share better resources, effectively solve the low utilization rate of university information network and the difficulty of equipment maintenance. Difficulties in information management and sharing.

\section{Current situation of domestic and foreign research}

Current situation of foreign research. In foreign countries, cloud computing has been highly recognized by educational institutions because of its great potential application value, and its application in the field of education has been paid more and more attention. Cloud computing has been used in daily life more and more widely. At home, although cloud computing started later than other countries, in 2008, Google teamed up with colleges and universities to launch cloud computing applications in the education industry. Several giants, such as Microsoft, have also partnered with 
many colleges and universities, and the first "cloud computing assistance" was launched in 2009. Advanced training in Teaching was organized by the China Educational Technology Association in Shanghai. After 2009, All over the country are dominated by colleges and universities, and they have begun to gradually apply cloud computing to assist teaching. What can be predicted is that cloud computing will become the mainstream information technology in colleges and universities in the next few years. It has effectively promoted the improvement of students' autonomous learning and classroom teaching, and has brought convenience to teaching and scientific research.

A Survey of Smart Campus and Cloud Computing. The concept of Smart Campus: Intelligent campus refers to the intelligent campus work, learning and life integration environment based on the Internet of things. This integrated environment takes various application service systems as the carrier, and fully integrates teaching, scientific research, management and campus life. Broadly speaking, cloud computing is the increase in Internet related services, the use and delivery mode, to provide the virtual and dynamic scalable resources through the Internet, it means that the computing power as a commodity circulation through the Internet. Infrastructure mode of cloud computing infrastructure can be divided into three categories: public cloud, private cloud and hybrid cloud. Public cloud public cloud computing refers to the third party provider by Internet for free or low price available to registered users can use the cloud, it usually consists of a service provider. The maintenance provider to provide services directly to external users through the infrastructure itself, external users although not in the true sense with cloud computing resources, but can enjoy all kinds of services provided by cloud network.

\section{Private cloud -- cloud base.}

The internal firewall facilities and hardware resources in enterprises or institutions, for the use of internal members, data center resources sharing between the various departments. Such as private cloud infrastructure management may be organization itself can also be third party, the location of the deployment can be made within the group can also be on the outside of the organization, as a private cloud is completely for a particular individual or organization and deployment of a separate, so do the reliable control of. in data / safety and quality of service are hybrid cloud hybrid cloud as the name suggests, is a mixed mode combination of public and private cloud two service mode. In this mode, private and public cloud is not lack of coordination, but presents. in terms of security to coordinate the work of the way: in private cloud computing storage, confidential data, non confidential data can use public cloud Service; in terms of cloud computing capability, we need to use private cloud processing business under normal demand, and use public cloud service when peak demand or computation and storage capacity are insufficient.

The concept of cloud computing: Cloud computing is an increase, use, and delivery model of Internet-based related services, often involving the provision of dynamically scalable and often virtual resources via the Internet. The cloud is a network. A metaphor for the Internet. In the past, the cloud was used to represent the telecommunications network, and later to represent the abstraction of the Internet and the underlying infrastructure. Therefore, cloud computing can even allow you to experience 10 tillion calculations per second. With such powerful computing power to simulate nuclear explosions, predict climate change and market trends, users can access data centers through computers, laptops, mobile phones, etc. Cloud computing combined with smart campus. Like the intelligent earth, the smart campus is inextricably linked to the Internet of things technology, the main goal of which is to connect the various devices and infrastructure in the campus network through the Internet of things technology. Provide an integrated environment of intelligence perception. In addition to the intelligent environment, the intelligent campus can also provide a basic support platform to provide storage, computing, sharing and other services for the upper services. Only in this way can we understand the wisdom of the intelligent campus. Cloud computing as a mature form of Internet operation, the earliest application in the field of education represents two aspects: on the one hand, cloud computing, on the other hand, cloud services, the introduction of these two aspects of cloud computing into the intelligent campus infrastructure platform, it provides the support for the 
characteristics of the intelligent campus and makes the wisdom characteristic of the intelligent campus more obvious and easy to be realized and prominent. Its primary goal is to provide an integrated intelligent environment of intelligence perception by connecting the various infrastructure in the campus network through the Internet of things devices. Intelligent campus must also provide a basic service support platform for all upper applications to provide storage, computing, sharing, and other services, and consider the realization of the advanced technology (such as big data processing technology, data mining technology), Only in this way can the wisdom of the campus be realized.

Design of Intelligent Campus Support Service Platform Based on Cloud Computing.

The overall goal of the construction of intelligent campus support service platform based on cloud computing is to provide support services by using cloud computing according to the current situation of information system construction in colleges and universities and the needs of the present stage. Help intelligent campus support service platform design and implementation. The specific objectives are to deploy cloud computing environment, build storage and computing clusters, obtain, It is a kind of service that obtains the perfect computer infrastructure resources in cloud computing through the Internet, mainly including various it and memory distributed in different geographical locations. Network bandwidth and other computer resources. The cloud storage service similar to the network disk on the network is a typical service in it. The typical has Amazon's elastic cloud and Apache's open source cloud computing framework. optimize the operation of cloud computing clusters, unify identity authentication services, design user authentication and single sign-on schemes, Can login all the system goals and upload to the upper layer. It service to achieve registration and provide the corresponding interface.

\section{The Overall Design of Intelligent Campus Support Service Platform.}

According to the cloud computing service model, the whole campus support service platform can adopt the cloud architecture. The framework is divided into three layers: the infrastructure layer: mainly is the formation of the resource pool to organize a large number of hardware resources, to provide support for the upper, including servers, storage, cyber source etc platform support layer: mainly provides the platform of software development environment or support services. Including storage, computing, resource query, user authentication service. The software application layer: call platform layer service support, to meet a variety of service system of campus application, provide specific application for campus users. Bus topology: The bus topology uses a channel as the transmission medium, and all stations are connected directly to the common transmission medium via the corresponding hardware interface. The public transmission medium is called the bus. The signal transmitted by any station is transmitted along the transmission medium and can be received by all other stations.

The advantages of the bus topology are as follows: the number of cables needed for the bus structure is small, the cable length is short, the bus is easy to be wired and maintained, the bus structure is simple, it is also a source of work, and it has higher reliability. The transmission rate is high, and it can be as high as $1 \sim 100$ ) it is easy to be expanded. Adding or reducing the number of users is more convenient, simple structure, easy networking, easy network expansion multiple nodes share a transmission channel, channel utilization. Design of distributed Storage Computing platform. In order to take into account, the development cost and the advanced nature of the platform, the design and development of it on the cloud computing framework is chosen because it has good encapsulation, exception handling, backup, etc. The function of load balance and extinction makes users pay more attention to the storage application and development of computers, and can support distributed storage very well, so it is fully capable of distributed computing. Design of Unified Identity Authentication platform. The Unified Identity Authentication platform is the access portal provided by the intelligent campus for the new and old systems. After the authentication of the portal site, the user enters the corresponding system through the corresponding link of the portal. It is responsible for providing the supporting services of user identity authentication and single sign-on. Design of Unified Authentication platform for Services. 
Transforms all types of Web services into standard unified interfaces in the campus network, where users access the corresponding interfaces to get the required services. And in it, The user is made up of the user and the server. The former uses the interface to apply for the required service. The latter can tell the system what service it can provide through the service registration and update application. For both users, it will review the application submitted, and after the examination, the corresponding items in the service directory database will be operated. Implementation and Evaluation of Campus Support Service platform based on Cloud Computing Intelligence. Platform Development Environment and Cluster deployment: Node configuration, Master-slave planning, Namenode and datanode, Ssh password free remote login configuration, cluster control, it master-slave setting. Implementation of Virtual Distributed Storage and Computing platform.

\section{Conclusion}

Cloud computing has been widely used in the field of education. This paper makes use of the advantages of cloud computing, through the research of campus users' needs and the analysis of cloud computing technology, introduces it into the construction of intelligent campus. This paper designs and implements a service platform for intelligent campus, which supports the realization of intelligent campus, and puts forward a set of design scheme, which improves the deficiency of cloud computing in practice, and verifies the feasibility of cloud computing in the construction of intelligent campus. In this paper, the intelligent campus support service platform has passed the test, with good practicability, compared with the traditional digital campus support platform, the intelligent campus support platform based on cloud computing has the following characteristics: using cloud architecture to further integrate software and hardware resources, and to achieve efficient and reliable computing power at the same time of low cost. With the rapid development and popularization of Internet, mobile Internet technology, personnel and resources in the university campus interaction is also undergoing profound changes, from simple human-computer interaction, the development between people and people, people and resources, various business systems interact with data transmission through a variety of mobile devices, smart equipment. Through the new technologies such as cloud computing, the integration of teaching, research, management and campus resources and application systems, and establishing a campus mode of intelligent service and management has become a major goal of information construction in universities. The wisdom of the campus itself has a huge data resources, each business system will produce a large amount of data constantly, each business system will be a lot of data exchange, the data is structured or unstructured, it is therefore stored the data center is very important. It can be said that data stored in data center is the application foundation of smart campus. Whether building a successful data center is the key factor to decide whether the smart campus is successful.

The information of colleges and universities started early, and the data centers of "smart campus" are mostly built on the basis of the original data center. With the deepening of education information construction, various hardware and software resources more and more wisdom in the campus, facing the smart campus increasingly complex application requirements, how to integrate resources, has become urgent to solve the problem of the wisdom of the campus to provide quality service on campus. On the basis of studying the advantages of cloud technology and the characteristics of cloud services, this paper designs a cloud computing based intelligent campus support service platform. It is the application of cloud computing in educational information. It integrates information into cloud services in many fields such as daily teaching and research activities in schools, so as to provide support for building a shared and efficient smart campus and realizing its intelligence characteristics. First of all, the paper analyzes the problems existing in the existing application system in smart campus, and determines the services provided by the platform and the level of service based on the needs survey of university users. The platform mainly provides distributed storage services, distributed computing services, user authentication services, single sign on services, Web services registration, and Web services look up it support services. Secondly, through the analysis of cloud computing and its related technologies, the process of using it services and the design of specific 
services are determined.

Cloud technology is used to support the massive data of intelligent campus, especially the accurate data basis for campus decision-making. Support services are more versatile and can be adapted to different environments, so they can be adapted to different campuses. However, due to the limitations of time, author level, hardware and other factors, it is still necessary to perfect and improve the intelligent campus support platform to make the intelligent campus support platform more perfect.

\section{Acknowledgements}

Youth project of social science fund of Jiangsu Province in 2015.

Research on the strategy and practice of the construction of smart campus under large data background (15JYC001).

\section{References}

[1] W.X. Based on Cloud Computing Design of Intelligent Campus Construction in Kunming first Middle School by stages. Analysis and Research [D] Kunming Yunnan University 2015.

[2] Q.T.H, K.Z, N.L. Education Informatization Development Transformation, from Digital Campus to Smart Campus [J] Chinese Audio-visual Education 2014.

[3] Research on the Application of Xu Xiaohua's Cloud Computing and Internet of things in the Construction of Intelligent Campus [J] Information Communication 2016.

[4] Research on the Construction of Intelligent Campus in higher Vocational Colleges in the era of Zhao Sijia Yin Ting big data [J] Information Systems Engineering 2016.

[5] Q.Z.Y. China Construction Information 2012.

[6] Analysis of Zhao Peihua's Intelligent Campus based on Cloud Computing Changzhou Institute of Information Technology 2012.

[7] X.H. Based on Cloud Computing Intelligence Campus Exploration [J] Primary and Middle School Audio-visual Education 2011.

[8] Wu handsome Zhejiang University based on Cloud Smart Campus [J] China Education Network 2010.

[9] Y.Z.Z. Discussion on the guarantee Mechanism of Intelligent Campus in Colleges and Universities [J] China's Management Informatization 2016.

[10] Design and implementation of Wang Rui's Intelligent Campus Service platform based on Cloud Computing [J] Wireless Interconnection Technology 2016. 